\title{
Quality and Quantity: How to Organize a Countrywide Genetic Counseling and Testing
}

\author{
Rita Katharina Schmutzler \\ Center for Familial Breast and Ovarian Cancer and Center for Integrated Oncology (CIO), University Hospital \\ Cologne, Cologne, Germany
}

\section{Keywords \\ Hereditary breast and ovarian cancer - Genetic counseling and testing - Structured and standardized care program . Evidence-generating care · Area coverage}

\begin{abstract}
Background: About $30 \%$ of all women with breast or ovarian cancer exhibit a family history of the disease. So far, the genetic cause could be deciphered in about $30 \%$ of these cases. The results demonstrate a high genetic heterogeneity, with high-risk and moderate-risk genes and low-risk variants contributing alone or in concert to the development of cancer. Furthermore, it has been shown that the genotype significantly determines the phenotype and that knowledge of the phenotype is as important as the genotype to offer adequate and risk-adapted prevention to persons at risk. For newly identified risk genes, however, the phenotype is not sufficiently characterized at first, and thus prevention measures are not sufficiently evaluated. Summary: The German Consortium for Hereditary Breast and Ovarian Cancer has developed a concept for collecting the missing data in the context of knowledge-generating care and at the same time ensuring care based on the best available knowledge. Core elements of this concept are: structured and standardized care, an outcome-oriented evaluation based on a comprehensive registry, networking with certified breast and gynecological cancer centers combined with regular training on state-of-theart care for doctors, and compilation of comprehensible patient information. This comprehensive concept has been incorporated into contracts for specialized care with health insurers and thus ensures nationwide care at the highest sci-
\end{abstract}

entific and clinical levels. Key Messages: This article describes how to implement a concept of evidence-generating care for risk-adjusted prevention in a nationwide health care system.

(c) 2021 The Author(s)

Published by S. Karger AG, Basel

\section{The Problem of Benefit Assessment of Genetic Tests}

With the increasing discovery of risk genes as heritable causes for the development of up to one third of a number of solid tumor diseases, most notably breast and colorectal cancer, a growing number of genetic tests are being offered and increasingly in the context of gene panel testing. With regard to breast and ovarian cancer, it is estimated that around $50 \%$ of the risk genes have now been identified. Disease penetrances vary widely, ranging from 20 to $80 \%$ for breast cancer and from 5 to $40 \%$ for ovarian cancer throughout life. Ongoing scientific investigations are constantly revealing new risk genes that are already being introduced into clinical diagnostics when only increased OR in case-control studies indicate an increase in risk. Since these OR are initially collected in risk-enriched collectives, they should by no means be considered as an approximation of relative risk, thus leading to misinterpretations.

According to established concepts for evaluating the evidence of genetic testing, the analytical validity, the clinical validity, and the clinical utility of the clinical measures based on the genetic findings have to be assessed [1]. Regarding analytical validity, the high rate of genetic alterations of unknown significance has to be considered in particular, as it accounts for a large proportion of genetic alterations identified in newly discovered risk genes and mercial purposes requires written permission. 
usually leads to uncertainty for both patients and physicians. This is because, unusually, there exists no gold standard for validation of such unclear variants. Rather, adequate analytical methods have to be identified and established in accompanying scientific projects, which may vary depending on the gene. These are, for example, evaluations based on large family collectives using likelihood models to calculate cosegregation, cooccurrence, and familial burden as well as functional testing systems [2-6]. With respect to clinical validity, questions regarding the prevalence of mutations in the presence of the disease and age-dependent penetrances are of primary concern. It should be noted at this point that, although information about a lifelong risk of disease is an important parameter from a health economic point of view, it is unsuitable for the decision-making of an affected person regarding the uptake of preventive measures. To illustrate, what does a risk of developing the disease by the age of 80 years indicate for the disease prevention of a 20 year old? Nothing! Therefore, it is important to communicate age-related disease rates, taking into account competing risks such as recurrence or secondary disease rates. These data, too, can only be collected by prospective studies of large cohorts for which international consortia have been formed beyond the national associations to generate the required number of cases (e.g., for BRCA1/2 [7] and for PALB2 [8]). Finally, clinical benefit must be substantiated by demonstrating that the preventive measures prompted by the genetic findings actually have an impact on the hard endpoints, i.e., morbidity, mortality, and quality of life. Since genetically defined subtypes have a specific phenotypic appearance, it should not automatically concluded that there is a benefit in the genetically defined subgroup from evidence of benefit of an intervention in the overall group of diseased individuals as, e.g., outlined in the position paper on risk-adjusted cancer screening of the German National Cancer Plan [9]. A clinical benefit of preventive measures has so far only been demonstrated for BRCA1/2-associated breast and ovarian cancer as outlined in, e.g., the German S3 guideline on breast cancer [10].

\section{Embedding Genetic Diagnostics in Knowledge- Generating Health Care}

The German Consortium for Hereditary Breast and Ovarian Cancer (GC-HBOC) focuses on closing this knowledge gap between genetic diagnostics and the clinical benefit of preventive measures based on it. The aim of the GC-HBOC is therefore to embed genetic diagnostics in an overall clinical concept for risk-adapted prevention of breast and ovarian cancer on the basis of a clinical benefit assessment. This has recently been outlined for the German health care situation [11] and is outlined here in more general terms.

The GC-HBOC was founded and funded in 1996 with the support of the German Cancer Aid after the discovery of the high-risk genes BRCA1 and BRCA2. Within the framework of this funding, it has linked genetic diagnostics to a clinical care concept from the very beginning. The question of the best possible approach to evidence generation arose from the very beginning. Several particularities had to be taken into account:

- If there is evidence of an increased risk of disease due to a positive gene finding, the acceptance of a prospectively randomized allocation of affected individuals to risk-adapted preventive care is low.

- This situation is further aggravated by the fact that genetic subtypes are rare and thus randomized prevention trials have to be scheduled many times longer than is usually the case for therapy trials. Projections assume several decades.

- For interventional and nonreversible preventive surgeries such as prophylactic bilateral mastectomy, randomization is not feasible according to prevailing ethical principles and it is not acceptable in the majority of cases.

In this situation, knowledge-generating care according to the recommendations of the German Cancer Society is a goal-oriented option. Therefore, the GC-HBOC, now consisting of 23 university centers, has initiated a prospective cohort study to generate the best possible evidence in this way. This implies documentation of the outcomes, which was realized starting in 2001 with the establishment of a database at the University of Leipzig. Initially, the focus was on the evaluation of analytical and clinical validity and later on extended to clinical utility. It is now complemented by a biobank at the University of Cologne.

Since 2010, the care concept has been supported by contracts with health insurance companies. In this context, a care network with certified breast and gynecological cancer centers has also be established to ensure nationwide care. In the meantime, more than 200 cancer centers throughout Germany have joined the network [12].

One of the first clinically relevant questions was the quality of the normatively defined inclusion criteria for genetic testing in a collective of more than 25,000 analyzed families [13], which thus represent reliably validated entrance criteria. Although the thresholds for offering genetic testing, which according to international consensus (e.g., NICE guideline) currently lie at a mutation detection rate of around $10 \%$, can and should be debated, collection of the evidence base for this is a sine qua non condition that is often forgotten in sometimes heated debates in this regard. 
Subsequently, the GC-HBOC in cooperation with its partners was able to extend the inclusion criteria to all TNBC patients aged $<50$ years and all ovarian cancer patients aged $<80$ years based on this threshold $[14,15]$.

\section{Structures and Processes for Implementing the Knowledge-Generating Care Concept}

The GC-HBOC has established the following structure for collaboration: the centers are represented by their center spokespersons in the center spokesperson committee, who in turn elect the coordinator who represents them externally. The center spokesperson committee usually meets once a year and takes all decisions of the consortium. Since the contract with the health insurance companies was established, a steering committee consisting of the center spokespersons and representatives of the contract health insurance companies was founded, in which data and results of the GC-HBOC are presented at regular intervals and, if necessary, the care concept is adapted. Subordinate to this are representatives of the core disciplines, i.e., clinicians, human geneticists, radiologists, and molecular geneticists, to which the centers each delegate 1-2 representatives and which are represented by an elected chairman. Meetings of the divisions are held 1-2 times per year. Results from the divisions are presented to the center spokespersons' committee and any consequences that can be derived from them are voted on there. The division chairs are also responsible for the evaluation of research projects and thus the release for a data query at the data center in Leipzig. For participation in international research projects, all centers receive a request with an opt-in option in advance.

The research projects are facilitated by the central database at the IMISE of the University of Leipzig (head of the database: PD Ch. Engel), in which extensive data sets are recorded in a quality-controlled manner on the basis of a standardized electronic remote documentation concept. To date, over 67,000 molecularly and clinically characterized families have been recruited. The IMISE is also responsible for providing methodological expertise in biometrics and clinical genetic epidemiology for scientific data analyses.

During the past year, the database has undergone a major reorganization. The HerediCaRe study [16], funded by the Federal Ministry of Education and Reseach (FMER) in the funding program Establishment of Model Registries for Health Services Research is currently establishing a registry for genotype-phenotype correlation and generation of prospective data. The goal is to evaluate hard clinical endpoints by linking the GC-HBOC registry to the existing clinical and epidemiological cancer registries. The GC-HBOC biobank established at the Cologne
Center since 2013, which now comprises more than 27,000 DNA samples, is the basis for national and international research projects.

Recently, the expert panel of the GC-HBOC for the clinical evaluation of genetic variants acquired a joint grant from the German Cancer Aid in the context of which the classification of genetic variants of unknown significance is systematized and accelerated by state-ofthe-art bioinformatic tools, functional test systems, and web interfaces. This is the basis for: (1) the recall system of the GC-HBOC, which allows recontact of patients in case of reclassification of a genetic variant via an automated processes and (2) consolidation of genetic data analysis by establishing uniform data formats.

\section{Accompanying Research Projects as an Immanent Component of Knowledge-Generating Care}

As already explained, clinical care in the GC-HBOC takes place within the framework of a knowledge-generating care concept. Knowledge generation requires accompanying scientific programs. To this end, the GCHBOC conducts research projects in the areas of: (1) genetic diagnostics, (2) clinical prevention (3) education and health literacy, (4) ELSI aspects, and (5) the development of new care concepts.

\section{Genetic Diagnostics}

As mentioned above, the discovery of breast cancer genes has not yet been completed and requires further genome-wide investigation. This necessitates newly discovered genes to be subjected to constant clinical evaluation with respect to the effectiveness of preventive measures. The GC-HBOC is member of the international consortium CIMBA for research of disease penetrance and phenotype in the hereditary burden and contributes to the discovery of new risk genes and their phenotyping [17-24].

\section{Clinical Prevention}

The GC-HBOC regularly evaluates the clinical results from the intensified screening program [25], which are used for the adaptation of the preventive measures in terms of an outcome-oriented evaluation; e.g., a narrowing of the screening program is currently underway after the evaluation revealed over-screening for women at moderate risk of breast cancer in young ages.

\section{Education and Health Literacy}

The GC-HBOC has established a modular training program for cooperation cancer centers, which includes a theoretical training course and a hands-on hospitation. This is obligatory for the conclusion of cooperation agreements and ensures uniform care in the network. Through 
this cooperation, it was possible to perform a joint evaluation within the cooperation partner network, which revealed that a proportion of about $30 \%$ fulfill the criteria for the offer of genetic testing [26]. In the future, the training concept is to be offered as a "blended learning tool" in cooperation with the German Cancer Society throughout Germany via a website and it is being funded by the Federal Ministry of Health (FMH). Furthermore, several third-party-funded projects (supported by the Innovation Fund, among others) are currently underway to improve the health literacy of the population in the field of genetic diagnostics and risk perception with the aim of offering validated patient decision aids and internetbased information, including apps.

\section{ELSI Aspects}

Based on the paper on risk-adapted screening in the National Cancer Plan of Germany, two interdisciplinary research projects on the ELSI aspects were conducted, i.e., MoreRisk (funded by the FMH) and SysCon (funded by the FMER). The results with suggestions for implementation and reimbursement were published in 2018 in a governance perspective [27].

\section{Development of New Care Concepts}

A paper on risk-adapted screening in the National Cancer Plan was the basis of an EU consensus paper on risk-adapted prevention in the context of a FMH grant (RiskAP) and the EU CanCon/iPAAC initiative taking into consideration the above mentioned key points [28].

\section{Rapid Patient Access to State-of-the-Art Prevention and Therapy Concepts}

The overall concept, based on an iterative process of gaining knowledge from genome research and transferring it into structured, standardized, networked, and quality-controlled clinical care, will make it possible to provide citizens with timely and effective access to the constantly increasing knowledge in the field of genomebased risk-adjusted prevention in a form that is also appropriate in terms of health economics.

In this way, added value can be created by knowledge generation through comprehensive evaluations, which can be further advanced in the future through AI evaluations of "real data" on genotype/phenotype correlations. The concept is representative and directly transferable to other hereditary tumor entities, which account for about one third of the other common solid tumors, i.e., prostate and colorectal cancer.

\section{Acknowledgement}

The author would like to thank the GC-HBOC for its excellent and determined cooperation and the self-help group BRCA-Netzwerk for the continuous and beneficial collaboration, both of which made the implementation possible.

\section{Conflict of Interest Statement}

The author has no conflict of interests to declare.

\section{Funding Sources}

This work was supported by the German cancer Aid for the establishment and genetic diagnostics of the consortium (grants 110837 and 70111850), the Federal Ministry of Education and Research for funding of the consortium registry for health services research and the compilation of a governance perspective on riskadjusted prevention (grants 01GP1407B and FKZ01GY1901), and the Federal Ministry of Health for funding a project on ELSI aspects and the compilation of a position paper on risk-adjusted prevention (grants 2515FSB401 and IIA5-2512FSB002).

\section{References}

1 Centers for Disease Control and Prevention. ACCE model process for evaluating genetic tests. 2010. [cited $2021 \mathrm{Feb} 3$ ]. Available from: https://www.cdc.gov/Genomics/Gtesting/ Acce/Index.Htm.

2 Goldgar DE, Easton DF, Deffenbaugh AM, Monteiro AN, Tavtigian SV, Couch FJ; Breast Cancer Information Core (BIC) Steering Committee. Integrated evaluation of DNA sequence variants of unknown clinical significance: application to BRCA1 and BRCA2. Am J Hum Genet. 2004 Oct;75(4):535-44.

3 Easton DF, Deffenbaugh AM, Pruss D, Frye C, Wenstrup RJ, Allen-Brady K, et al. A systematic genetic assessment of 1,433 sequence variants of unknown clinical significance in the BRCA 1 and BRCA 2 breast cancer-predisposition genes. Am J Hum Genet. 2007 Nov; 81(5):873-83.

4 Kalb R, Neveling K, Hoehn H, Schneider H, Linka Y, Batish SD, et al. Hypomorphic mutations in the gene encoding a key Fanconi anemia protein, FANCD2, sustain a significant group of FA-D2 patients with severe phenotype. Am J Hum Genet. 2007 May;80(5):895-910.

5 Reid S, Schindler D, Hanenberg H, Barker K, Hanks S, Kalb R, et al. Biallelic mutations in PALB2 cause Fanconi anemia subtype FA-N and predispose to childhood cancer. Nat Genet. $2007 \mathrm{Feb} ; 39(2): 162-4$.

6 Goldgar DE, Easton DF, Byrnes GB, Spurdle $\mathrm{AB}$, Iversen ES, Greenblatt MS; IARC Unclas- sified Genetic Variants Working Group. Genetic evidence and integration of various data sources for classifying uncertain variants into a single model. Hum Mutat. 2008 Nov;29(11): 1265-72.

7 Kuchenbaecker KB, Hopper JL, Barnes DR, Phillips KA, Mooij TM, Roos-Blom MJ, et al.; BRCA1 and BRCA2 Cohort Consortium. Risks of Breast, Ovarian, and Contralateral Breast Cancer for BRCA1 and BRCA2 Mutation Carriers. JAMA. 2017 Jun;317(23):2402-16.

8 Yang X, Leslie G, Doroszuk A, Schneider S, Allen J, Decker B, et al. Cancer Risks Associated With Germline PALB2 Pathogenic Variants: An International Study of 524 Families. J Clin Oncol. 2020 Mar;38(7):674-85. 
9 Schmutzler RK, Dietz D, Jöckel KH, Greinert R, Hoffmann W, Riemann J, et al. Risk-adjusted cancer screening. 2011. [cited $2021 \mathrm{Feb}$ 3]. Available from: https://www.bundesgesundheitsministerium.de/fileadmin/Dateien/3_Downloads/N/Nationaler_Krebsplan/ Risk_adjusted_screening_for_cancer.pdf.

10 Deutsche Krebsgesellschaft. Leitlinienprogramm Onkologie AW. S3-Leitlinie Früherkennung, Diagnose, Therapie und Nachsorge des Mammakarzinoms. 2020. [cited 2021 Feb 3]. Available from: https://www. Leitlinienprogramm-Onkologie.De/Fileadmin/User_Upload/Downloads/Leitlinien/ Mammakarzinom_4_0/Version 4.3/Ll Mammakarzinom_Langversion_4.3.Pdf.

11 Schmutzler RK. Risikoadaptierte Prävention bei familiär bedingtem Brust- und Eierstockkrebs. Forum. 2021;36(1):16-20.

12 Deutsches Konsortium Familiärer Brust- und Eierstockkrebs. Universitätsklinikum Köln. Zentren des Konsortiums und ihre Kooperationspartner. 2021. [cited 2020 Nov 16]. Available from: https://www.konsortium-familiaerer-brustkrebs.de/das-konsortium/kooperationspartner/.

13 Kast K, Rhiem K, Wappenschmidt B, Hahnen E, Hauke J, Bluemcke B, et al.; German Consortium for Hereditary Breast and Ovarian Cancer (GC-HBOC). Prevalence of BRCA1/2 germline mutations in 21401 families with breast and ovarian cancer. J Med Genet. 2016 Jul;53(7):465-71.

14 Harter P, Hauke J, Heitz F, Reuss A, Kommoss S, Marmé F, et al. Prevalence of deleterious germline variants in risk genes including BRCA $1 / 2$ in consecutive ovarian cancer patients (AGO-TR-1). PLoS One. 2017 Oct; 12(10):e0186043.

15 Engel C, Rhiem K, Hahnen E, Loibl S, Weber KE, Seiler S, et al.; German Consortium for Hereditary Breast and Ovarian Cancer (GCHBOC). Prevalence of pathogenic BRCA1/2 germline mutations among 802 women with unilateral triple-negative breast cancer with- out family cancer history. BMC Cancer. 2018 Mar;18(1):265.

16 Deutsches Konsortium Familiärer Brust- und Eierstockkrebs. Universitätsklinikum Köln. Deutsches Konsortium Familiärer Brust- und Eierstockkrebs. 2021. [cited 2020 Nov 16]. Available from: https://www.konsortium-familiaerer-brustkrebs.de/.

17 Meindl A, Hellebrand H, Wiek C, Erven V, Wappenschmidt B, Niederacher D, et al. Germline mutations in breast and ovarian cancer pedigrees establish RAD51C as a human cancer susceptibility gene. Nat Genet. 2010 May;42(5):410-4.

18 Michailidou K, Lindström S, Dennis J, Beesley J, Hui S, Kar S, et al.; NBCS Collaborators; ABCTB Investigators; ConFab/AOCS Investigators. Association analysis identifies 65 new breast cancer risk loci. Nature. 2017 Nov; 551(7678):92-4.

19 Milne RL, Kuchenbaecker KB, Michailidou K, Beesley J, Kar S, Lindström S, et al.; ABCTB Investigators; EMBRACE; GEMO Study Collaborators; HEBON; kConFab/AOCS Investigators; NBSC Collaborators. Identification of ten variants associated with risk of estrogen-receptor-negative breast cancer. Nat Genet. 2017 Dec;49(12):1767-78.

20 Wu L, Shi W, Long J, Guo X, Michailidou K, Beesley J, et al.; NBCS Collaborators; kConFab/ AOCS Investigators. A transcriptome-wide association study of 229,000 women identifies new candidate susceptibility genes for breast cancer. Nat Genet. 2018 Jul;50(7):968-78.

21 Ferreira MA, Gamazon ER, Al-Ejeh F, Aittomäki K, Andrulis IL, Anton-Culver H, et al.; EMBRACE Collaborators; GC-HBOC Study Collaborators; GEMO Study Collaborators; ABCTB Investigators; HEBON Investigators; BCFR Investigators. Genome-wide association and transcriptome studies identify target genes and risk loci for breast cancer. Nat Commun. 2019 Apr;10(1):1741.

22 Fachal L, Aschard H, Beesley J, Barnes DR, Allen J, Kar S, et al.; GEMO Study Collabo- rators; EMBRACE Collaborators; KConFab Investigators; $\mathrm{HEBON}$ Investigators; ABCTB Investigators. Fine-mapping of 150 breast cancer risk regions identifies 191 likely target genes. Nat Genet. 2020 Jan;52(1): 56-73.

23 Yang X, Song H, Leslie G, Engel C, Hahnen E, Auber B, et al. Ovarian and breast cancer risks associated with pathogenic variants in RAD51C and RAD51D. J Natl Cancer Inst. 2020 Dec;112(12):1242-50.

24 Zhang H, Ahearn TU, Lecarpentier J, Barnes D, Beesley J, Qi G, et al.; kConFab Investigators; ABCTB Investigators; EMBRACE Study; GEMO Study Collaborators. Genome-wide association study identifies 32 novel breast cancer susceptibility loci from overall and subtype-specific analyses. Nat Genet. 2020 Jun;52(6):572-81.

25 Bick U, Engel C, Krug B, Heindel W, Fallenberg EM, Rhiem K, et al.; German Consortium for Hereditary Breast and Ovarian Cancer (GC-HBOC). High-risk breast cancer surveillance with MRI: 10-year experience from the German consortium for hereditary breast and ovarian cancer. Breast Cancer Res Treat. 2019 May;175(1):217-28

26 Rhiem K, Bücker-Nott HJ, Hellmich M, Fischer H, Ataseven B, Dittmer-Grabowski C, et al. Benchmarking of a checklist for the identification of familial risk for breast and ovarian cancers in a prospective cohort. Breast J. 2019 May;25(3):455-60.

27 Meier F, Harney A, Rhiem K, Neumann A, Neusser S, Braun M, et al. Risikoadaptierte Prävention. Berlin: Springer VS; 2018. [cited 2020 Nov 16]. Available from: https://www. springer.com/de/book/9783658208004.

28 Schmutzler RK, Schmitz-Luhn B, Borisch B, Devilee P, Eccles D, Hall P, et al. Risk-adjusted cancer screening and prevention (RiskAP): complementing screening for early disease detection by a learning screening based on risk factors. Breast Care. 2021; accepted for publication. 